\title{
MONOTONICITY FORMULAE AND F-STRESS ENERGY
}

\author{
Nour Elhouda DJAA ${ }^{1}$, Ahmed MOHAMED CHERIF $^{2}$ and \\ Kaddour ZEGGA*,3
}

\begin{abstract}
The goal of this work is the application of the f-stress energy of differential forms to study the generalized monotonicity formulae and generalized vanishing theorems. We obtain some generalized monotonicity formulas for $p$-forms $\omega \in A^{p}(\xi)$, which satisfy the generalized $f$-conservation laws, with $f \in C^{\infty}(M \times \mathbb{R})$ satisfying some conditions.

2000 Mathematics Subject Classification: 53A45, 53C20, 58E20 .

Key words: $f$-stree-energy, $f$-conservation law, vanishing theorem.
\end{abstract}

\section{Introduction}

In 1980, Baird and Eells [2] introduced the stress-energy tensor for maps between Riemannian manifolds, which unifies various results on harmonic maps. Following [2], Sealey [13] introduced the stress-energy tensor for p-forms with values in vector bundles and established some vanishing theorems for harmonic p-forms. Since then, the stress-energy tensors have become a useful tool for investigating the energy behavior of vector bundle valued p-forms in various problems. In [7] the authors presented a unified method to establish monotonicity formulae for p-forms with values in vector bundles by means of the stress-energy tensors of various energy functionals in geometry and physics.

Recently in 2010, M. Djaa and all introduced the notion of f-harmonic and f-stress energy [11], [4] and studied by many authors Chiang [3], Y.L. Ou [12], S. Feng [8], W.J. Lu [10] and others.

The goal of this work is the application of the f-stress energy of differential forms to study the generalized monotonocity formulae and generalized vanishing

\footnotetext{
${ }^{1}$, Faculty of Sciences and Technology, Relizane University , e-mail: Djaanour@gmail.com

${ }^{2}$ Department of Mathematics, Mascara University, e-mail: Ahmed29cherif@gmail.com

3* Corresponding author, Department of Mathematics, Mascara University, e-mail: K29.zegga@gmail.com
} 
theorem (Theorem 3.1, Theorem 3.3 and Theorem 3.4).

Let $(M, g)$ be a Riemannian manifold and $\xi: E \longrightarrow M$ be a smooth Riemannian vector bundle over $M$. Set:

$$
A^{p}(\xi)=\Lambda^{p} T^{*} M \otimes E
$$

the space of smooth $p$-froms on $M$ with values in the vector bundle $\xi: E \longrightarrow M$.

For a linear connection $\nabla^{E}$ on $E$ we define the covariant derivative on $A^{p}(\xi)$ by

$$
\left(\nabla_{X} \omega\right)\left(X_{1}, \ldots . ., X_{p}\right)=\nabla_{X}^{E} \omega\left(X_{1}, \ldots ., X_{p}\right)-\sum_{i=1}^{p} \omega\left(X_{1}, . ., \nabla_{X} X_{i}, \ldots, X_{p}\right)
$$

The exterior covariant differentiation $d^{\nabla}: A^{p}(\xi) \longrightarrow A^{p+1}(\xi)$ relative to the connection $\nabla^{E}$ is defined by

$$
\left(d^{\nabla} \omega\right)\left(X_{1}, X_{2}, . ., X_{p+1}\right)=\sum_{i=1}^{p+1}(-1)^{i+1}\left(\nabla_{X_{i}} \omega\right)\left(X_{1}, X_{2}, . ., \hat{X}_{i}, . ., X_{p+1}\right),
$$

where the symbols covered by ${ }^{\wedge}$ are omitted and $X_{i} \in \Gamma(T M)$ for $i=1, \ldots, p+1$. The codifferential operator $\delta^{\nabla}: A^{p}(\xi) \longrightarrow A^{p-1}(\xi)$ characterized as the adjoint of $d^{\nabla}$ is defined by

$$
\left(\delta^{\nabla} \omega\right)\left(X_{1}, X_{2}, . ., X_{p-1}\right)=-\sum_{i=1}^{m}\left(\nabla_{e_{i}} \omega\right)\left(e_{i}, X_{1}, X_{2}, . ., X_{p-1}\right) .
$$

Here $\left\{e_{1}, e_{2}, . ., e_{m}\right\}$ is a local frame field on $(M, g)$.

For $\omega \in A^{p}(\xi)$, we define the generalized $f$-energy functional of $\omega$ as follows:

$$
E_{f}(\omega)=\int_{M} f\left(x, \frac{|\omega|^{2}}{2}\right) v_{g}
$$

where $f: M \times \mathbb{R} \longrightarrow \mathbb{R},(x, s) \longmapsto f(x, s)$ is a smooth function such that $f(x, 0)=$ 0 , for all $x \in M$ and $f(x, s)>0$, for all $(x, s) \in M \times \mathbb{R}_{+}^{*}$. Here:

$$
|\omega|^{2}=<\omega, \omega>=\sum_{i_{1}<i_{2}<. .<i_{p}}<\omega\left(e_{i_{1}}, e_{i_{2}}, . ., e_{i_{p}}\right), \omega\left(e_{i_{1}}, e_{i_{2}}, . ., e_{i_{p}}\right)>_{E}
$$

If $f(x, s)=\tilde{f}(x) s, \forall(x, s) \in M \times \mathbb{R}$ we deduce the energy functional of $\omega$ defined in [9], where $\tilde{f}: M \longrightarrow(0, \infty)$ is a smooth function. 


\section{Generalized $f$-stress energy tensor}

If $(g)_{t}$ is a smooth 1-parameter family of metrics with $g_{0}=g$, then the variation $\delta g=\left.\frac{\partial g}{\partial t}\right|_{t=0}$ is a smooth symmetric tensor on $M$. We have:

Proposition 1. Let $(M, g)$ be a Riemannian manifold and $\xi: E \longrightarrow M$ be a smooth Riemannian vector bundle over $M$ with a metric compatible connection $\nabla^{E}$ and $\omega \in A^{p}(\xi)$, then

$$
\left.\frac{d}{d t} E_{f}(\omega)\right|_{t=0}=\frac{1}{2} \int_{M}<S_{f}(\omega), \delta g>v_{g}
$$

where:

$$
S_{f}(\omega)=f_{\omega} g-f_{\omega}^{\prime} \omega \odot \omega
$$

is called generalized $f$-stress energy tensor for $\omega, f_{\omega} \in C^{\infty}(M)$ is a smooth function defined by:

$$
f_{\omega}(x)=f\left(x, \frac{|\omega|^{2}}{2}\right), \quad \forall x \in M
$$

and $f_{\omega}^{\prime} \in C^{\infty}(M)$ is a smooth function defined by:

$$
f_{\omega}^{\prime}(x)=\frac{\partial f}{\partial s}\left(x, \frac{|\omega|^{2}}{2}\right), \quad \forall x \in M .
$$

Proof. We have:

$$
\begin{aligned}
\left.\frac{d}{d t} E_{f}(\omega)\right|_{t=0} & =\int_{M} \frac{\partial}{\partial t}\left[f\left(x, \frac{|\omega|^{2}}{2}\right) v_{g_{t}}\right]_{t=0} \\
& =\int_{M}\left[\frac{\partial}{\partial t}\left(f\left(x, \frac{|\omega|^{2}}{2}\right)\right) v_{g_{t}}+f\left(x, \frac{|\omega|^{2}}{2}\right) \frac{\partial}{\partial t}\left(v_{g_{t}}\right)\right]_{t=0} \\
& =\int_{M}\left[\frac{1}{2} \frac{\partial}{\partial t}\left(\frac{|\omega|^{2}}{2}\right) \frac{\partial f}{\partial s}\left(x, \frac{|\omega|^{2}}{2}\right) v_{g_{t}}+f\left(x, \frac{|\omega|^{2}}{2}\right) \frac{\partial}{\partial t}\left(v_{g_{t}}\right)\right]_{t=0}
\end{aligned}
$$

From [1], we know that:

$$
\left.\frac{\partial}{\partial t}\left(\frac{|\omega|^{2}}{2}\right)\right|_{t=0}=-<\omega \odot \omega, \delta g>,
$$

and:

$$
\left.\frac{\partial}{\partial t}\left(v_{g_{t}}\right)\right|_{t=0}=\frac{1}{2}<g, \delta g>v_{g}
$$

so that

$$
\begin{aligned}
\left.\frac{d}{d t} E_{f}(\omega)\right|_{t=0} & =\frac{1}{2} \int_{M}<f_{\omega} g-f^{\prime} \omega \odot \omega, \delta g>v_{g} \\
& =\frac{1}{2} \int_{M}<S_{f}(\omega), \delta g>v_{g} .
\end{aligned}
$$


Proposition 2. Let $(M, g)$ be a Riemannian manifold and $\xi: E \longrightarrow M$ be a smooth Riemannian vector bundle over $M$ with a metric compatible connection $\nabla^{E}, f: M \times \mathbb{R} \longrightarrow(0, \infty),(x, s) \longmapsto f(x, s)$ a smooth function and $\omega \in A^{p}(\xi)$, then:

$$
\begin{aligned}
\left(\operatorname{div} S_{f}(\omega)\right)(X)= & X(f)_{\omega}-<i_{\operatorname{grad} f_{\omega}^{\prime}} \omega, i_{X} \omega> \\
& +f_{\omega}^{\prime}\left[<\delta^{\nabla} \omega, i_{X} \omega>+<i_{X} d^{\nabla} \omega, \omega>\right],
\end{aligned}
$$

for all $X \in \Gamma(T M)$, where $X(f)_{\omega} \in C^{\infty}(M)$ is a smooth function defined by:

$$
X(f)_{\omega}(x)=X(f)\left(x, \frac{|\omega|^{2}}{2}\right), \quad \forall x \in M .
$$

Proof. Let $\left\{e_{1}, . ., e_{m}\right\}$ be an orthonormal frame on $(M, g)$, such that at $x \in M$, $\nabla_{e_{i}} e_{j}=0$. By using (4), we have:

$$
\begin{aligned}
\left(\operatorname{div} S_{f}(\omega)\right)(X)= & \left.\left.\sum_{i=1}^{m}\left[\nabla_{e_{i}} S_{f}(\omega)\right)\left(e_{i}, X\right)-S_{f}(\omega)\right)\left(e_{i}, \nabla_{e_{i}} X\right)\right] \\
= & \sum_{i=1}^{m} \nabla_{e_{i}}\left[f_{\omega} g\left(e_{i}, X\right)-f_{\omega}^{\prime} \omega \odot \omega\left(e_{i}, X\right)\right] \\
& -\sum_{i=1}^{m}\left[f_{\omega} g\left(\nabla_{e_{i}} X, e_{i}\right)+f_{\omega}^{\prime} \cdot \omega \odot \omega\left(\nabla_{e_{i}} X, e_{i}\right)\right] \\
= & d f_{\omega}(X)-<i_{\operatorname{grad} f_{\omega}^{\prime}} \omega, i_{X} \omega> \\
& -\sum_{i=1}^{m} f_{\omega}^{\prime}\left[\nabla_{e_{i}}<i_{e_{i}} \omega, i_{X} \omega>-<i_{e_{i}} \omega, i_{\nabla_{i}} X \omega\right] .
\end{aligned}
$$

The first term in the right side of $(6)$ is

$$
\begin{aligned}
d f_{\omega}(X) & =X\left(f_{\omega}\right) \\
& =X\left(f\left(x, \frac{|\omega|^{2}}{2}\right)\right) \\
& =X(f)_{\omega}+X\left(\frac{|\omega|^{2}}{2}\right) f_{\omega}^{\prime} .
\end{aligned}
$$

From the Lemma (1.2) in ([9]), we deduce that:

$$
\begin{aligned}
X\left(\frac{|\omega|^{2}}{2}\right)= & \sum_{j_{1}<. .<j_{p-1}, i}<\omega\left(e_{i}, e_{j_{1}}, . ., e_{j_{p-1}}\right),\left(\nabla_{e_{i}} \omega\right)\left(X, e_{j_{1}}, . ., e_{j_{p-1}}\right)> \\
& +<i_{X} d^{\nabla} \omega, \omega>,
\end{aligned}
$$

so that:

$$
\begin{aligned}
d f_{\omega}(X)= & X(f)_{\omega}+f_{\omega}^{\prime}<i_{X} d^{\nabla} \omega, \omega> \\
& +f_{\omega}^{\prime} \sum_{j_{1}<j_{2}<. .<j_{p-1}, i}<\omega\left(e_{i}, e_{j_{1}}, . ., e_{j_{p-1}}\right),\left(\nabla_{e_{i}} \omega\right)\left(X, e_{j_{1}}, . ., e_{j_{p-1}}\right)>.
\end{aligned}
$$


for the third term in the right side of (6), we have:

$$
\begin{aligned}
<\delta^{\nabla} \omega, i_{X} \omega>= & \delta \nabla \omega\left(e_{i_{1}}, . ., e_{i_{p-1}}\right), \omega\left(X, e_{i_{1}}, . ., e_{i_{p-1}}\right)> \\
= & -<\sum_{i=1}^{m}\left(\nabla_{e_{i}} \omega\right)\left(e_{i}, e_{j_{1}}, . ., e_{j_{p-1}}\right), \omega\left(X, e_{j_{1}}, . ., e_{j_{p-1}}\right)> \\
= & -<\sum_{i=1}^{m} \nabla_{e_{i}} \omega\left(e_{i}, e_{j_{1}}, . ., e_{j_{p-1}}\right), \omega\left(X, e_{j_{1}}, . ., e_{j_{p-1}}\right)> \\
= & -\sum_{i=1}^{m} \nabla_{e_{i}}<\omega\left(e_{i}, e_{j_{1}}, . ., e_{j_{p-1}}\right), \omega\left(X, e_{j_{1}}, . ., e_{j_{p-1}}\right)> \\
& +\sum_{j_{1}<j_{2}<. .<j_{p-1}, i}<\omega\left(e_{i}, e_{j_{1}}, . ., e_{j_{p-1}}\right), \nabla_{e_{i}} \omega\left(X, e_{j_{1}}, . ., e_{j_{p-1}}\right)> \\
= & -\sum_{i=1}^{m} \nabla_{e_{i}}<i_{e_{i}} \omega, i_{X} \omega> \\
& +\sum_{j_{1}<. .<j_{p-1}, i}^{m}<i_{e_{i}} \omega,\left(\nabla_{e_{i}} \omega\right)\left(X, e_{j_{1}}, . ., e_{j_{p-1}}\right)+\omega\left(\nabla_{e_{i}} X, e_{j_{1}}, . ., e_{j_{p-1}}\right)> \\
= & \left.-\sum_{i=1}^{m}\left[\nabla_{e_{i}}<i_{e_{i}} \omega, i_{X} \omega>-<i_{e_{i}} \omega, i_{\nabla_{e_{i}} X} \omega\right)>\right] \\
& +\sum_{j_{1}<j_{2}<. .<j_{p-1}, i}<\omega\left(e_{i}, e_{j_{1}}, . ., e_{j_{p-1}}\right), \nabla_{e_{i}} \omega\left(X, e_{j_{1}}, . ., e_{j_{p-1}}\right)>,
\end{aligned}
$$

that is:

$$
\begin{gathered}
\left.\sum_{i=1}^{m}\left[\nabla_{e_{i}}<i_{e_{i}} \omega, i_{X} \omega>-<i_{e_{i}} \omega, i_{\nabla_{e_{i}} X} \omega\right)>\right]= \\
\sum_{j_{1}<. .<j_{p-1}, i}<\omega\left(e_{i}, e_{j_{1}}, . ., e_{j_{p-1}}\right), \nabla_{e_{i}} \omega\left(X, e_{j_{1}}, . ., e_{j_{p-1}}\right)>-<\delta^{\nabla} \omega, i_{X} \omega>.
\end{gathered}
$$

Finally, by replacing (8) and (9) in (6), we obtain the result of the proposition (2).

Definition 1. $\omega \in A^{p}(\xi)(p \geq 1)$ is said to satisfy a generalized $f$-conservation law if it satisfies the following equation:

$$
\left(\operatorname{div} S_{f}(\omega)\right)(X)=X(f)_{\omega}
$$

By applying $T=S_{f, \omega}$ in (7) (see [9]), we have:

$$
\int_{\partial D} S_{f, \omega}(X, \nu) d s_{g}=\int_{D}\left[\left\langle S_{f, \omega}, \frac{1}{2} L_{X} g\right\rangle+X(f)_{\omega}\right] d v_{g} .
$$

Let $\Gamma_{0}(T M)$ be a subset of $\Gamma(T M)$ consisting of all elements with compact supports contained in the interior of $M$. 
Definition 2. $\omega \in A^{p}(\xi)(p \geq 1)$ is said to satisfy a generalized integral $f$ conservation law if it satisfies the following equation:

$$
\int_{M}\left(\operatorname{div} S_{f, \omega}\right)(X) d v_{g}=\int_{M} X(f)_{\omega} d v_{g}
$$

for any $X \in \Gamma_{0}(T M)$.

From the equation (7) in [9], we have:

$$
\int_{M}\left[\left\langle T, \frac{1}{2} L_{X} g\right\rangle+(\operatorname{div} T)(X)\right] d v_{g}=0,
$$

for any $X \in \Gamma_{0}(T M)$. From Definition (2) and equation (11), we have:

$$
\int_{M}\left[\left\langle S_{f, \omega}, \frac{1}{2} L_{X} g\right\rangle+X(f)_{\omega}\right] d v_{g}=0,
$$

for any $X \in \Gamma_{0}(T M)$ and $\omega \in A^{p}(\xi)(p \geq 1)$ satisfies the generalized integral $f$-conservation law.

\section{Monotonicity Formulas and Vanishing Theorems}

Let $\left(M, g_{0}\right)$ be a complete Riemannian manifold with a pole $x_{0}$. Denote by $r(x)$ the $g_{0}$ distance function relative to the pole $x_{0}$, that is $r(x)=\operatorname{dis}_{g_{0}}\left(x, x_{0}\right)$. Set:

$$
B(r)=\left\{x \in M^{m}: r(x) \leq r\right\} .
$$

It is known that $\frac{\partial}{\partial r}$ is always an eigenvector of $\operatorname{Hess}_{g_{0}}\left(r^{2}\right)$ associated to eigenvalue 2. Denote by $\lambda_{\max }$ (resp. $\lambda_{\min }$ ) the maximum (resp. minimal) eigenvalues of $\operatorname{Hess}_{g_{0}}\left(r^{2}\right)-2 d r \otimes d r$ at each point of $M-\left\{x_{0}\right\}$.

From now on, we suppose that $\omega \in A^{p}(\xi)$ satisfies the generalized $f$-conservation law and $\xi: E \longrightarrow(M, g)$ is a smooth Riemannian vector bundle over $(M, g)$ where $g=\varphi^{2} g_{0}, 0<\varphi \in \mathrm{C}^{\infty}(M)$. Clearly, the vector field $\nu=\varphi^{-1} \frac{\partial}{\partial r}$ is an outer normal vector field along $\partial B(r) \subset(M, g)$. We assume the following conditions for $\varphi$ :

$\left(\varphi_{1}\right) \frac{\partial \log \varphi}{\partial r} \geq 0$

$\left(\varphi_{2}\right)$ there is a constant $C_{0}>0$ such that:

$$
(m-2 p) r \frac{\partial \log \varphi}{\partial r}+\frac{m-1}{2} \lambda_{\min }+1-p \max \left(2, \lambda_{\max }\right) \geq C_{0},
$$

now we set $\mu=\sup _{M \times \mathbb{R}_{+}^{*}} r\left|\frac{\partial \log f}{\partial r}\right|<\infty$.

Theorem 1. Suppose that $\omega \in A^{p}(\xi)(p \geq 1)$ satisfies the generalized $f$-conservaion law and $\xi: E \longrightarrow(M, g)$ is a smooth Riemannian vector bundle over $\left(M, \varphi^{2} g_{0}\right)$. If $C_{0}-\mu>0, \varphi$ satisfies $\left(\varphi_{1}\right),\left(\varphi_{2}\right)$ with $s \frac{\partial f}{\partial s} \leq f$, and $\frac{\partial f}{\partial s} \geq 0$, then

$$
\frac{\int_{B\left(\rho_{1}\right)} f_{\omega} d v_{g}}{\rho_{1}^{C_{0}-\mu}} \leq \frac{\int_{B\left(\rho_{2}\right)} f_{\omega} d v_{g}}{\rho_{2}^{C_{0}-\mu}},
$$

for any $0<\rho_{1}<\rho_{2}$ 
Proof. We take $D=B(r)$ and $X=r \frac{\partial}{\partial r}=\frac{1}{2} \nabla^{0} r^{2}$, where $\nabla^{0}$ denotes the covariant derivative determined by $g_{0}$. By a direct computation, we have :

$$
\begin{aligned}
\left\langle S_{f, \omega}, \frac{1}{2} L_{X}(g)\right\rangle & =\left\langle S_{f, \omega}, r \frac{\partial \log \varphi}{\partial r} g+\frac{1}{2} \varphi^{2} L_{X}\left(g_{0}\right)\right\rangle \\
& =r \frac{\partial \log \varphi}{\partial r}\left\langle S_{f, \omega}, g\right\rangle+\frac{1}{2} \varphi^{2}\left\langle S_{f, \omega}, \text { Hess }_{g_{0}}\left(r^{2}\right)\right\rangle .
\end{aligned}
$$

Let $\left\{e_{i}\right\}_{i=1}^{m}$ be an orthonormal basis with respect to $g_{0}$ and $e_{m}=\frac{\partial}{\partial r}$. We may assume that $\operatorname{Hess}_{g_{0}}\left(r^{2}\right)$ becomes a diagonal matrix with respect to $\left\{e_{i}\right\}$, then $\left\{\tilde{e}_{i}=\varphi^{-1} e_{i}\right\}$ is an orthonormal basis with respect to $g$. For the first term of (13):

$$
\begin{aligned}
\left\langle S_{f, \omega}, g\right\rangle & =\sum_{i, j=1}^{m} S_{f, \omega}\left(\tilde{e_{i}}, \tilde{e_{j}}\right) g\left(\tilde{e_{i}}, \tilde{e_{j}}\right) \\
& =\sum_{i, j=1}^{m}\left[f_{\omega} g\left(\tilde{e_{i}}, \tilde{e_{j}}\right) g\left(\tilde{e_{i}}, \tilde{e_{j}}\right)-f_{\omega}^{\prime}(\omega \odot \omega)\left(\tilde{e_{i}}, \tilde{e_{j}}\right) g\left(\tilde{e_{i}}, \tilde{e_{j}}\right)\right] \\
& =m f_{\omega}-f_{\omega}^{\prime} \sum_{i=1}^{m}\left\langle i_{\tilde{e_{i}}} \omega, i_{\tilde{e_{i}}} \omega>\right. \\
& =m f_{\omega}-f_{\omega}^{\prime} p|\omega|^{2} .
\end{aligned}
$$

For the second term of (13), we have:

$$
\begin{aligned}
\frac{1}{2} \varphi^{2}\left\langle S_{f, \omega}, \text { Hess }_{g_{0}}\left(r^{2}\right)\right\rangle= & \frac{1}{2} \varphi^{2} \sum_{i, j=1}^{m} S_{f, \omega}\left(\tilde{e_{i}}, \tilde{e_{j}}\right) \text { Hess }_{g_{0}}\left(r^{2}\right)\left(\tilde{e}_{i}, \tilde{e_{j}}\right) \\
= & \frac{1}{2} \varphi^{2} \sum_{i, j=1}^{m}\left[f_{\omega} g\left(\tilde{e_{i}}, \tilde{e_{j}}\right) \operatorname{Hess}_{g_{0}}\left(r^{2}\right)\left(\tilde{e_{i}}, \tilde{e_{j}}\right)\right. \\
& \left.-f_{\omega}^{\prime}(\omega \odot \omega)\left(\tilde{e_{i}}, \tilde{e_{j}}\right) \operatorname{Hess}_{g_{0}}\left(r^{2}\right)\left(\tilde{e_{i}}, \tilde{e_{j}}\right)\right] \\
= & \frac{1}{2} f_{\omega} \sum_{i=1}^{m} \operatorname{Hess}_{g_{0}}\left(r^{2}\right)\left(e_{i}, e_{i}\right) \\
& -\frac{1}{2} f_{\omega}^{\prime} \sum_{i=1}^{m}<i_{\tilde{e_{i}}} \omega, i_{\tilde{e_{i}}} \omega>\operatorname{Hess}_{g_{0}}\left(r^{2}\right)\left(e_{i}, e_{i}\right) \\
\geq & \frac{1}{2} f_{\omega}\left[(m-1) \lambda_{\min }+2\right]-\frac{1}{2} \max \left(2, \lambda_{\max }\right) f_{\omega}^{\prime} p|\omega|^{2} .
\end{aligned}
$$

From (13), (14), (15), $\left(\varphi_{1}\right)$ and $\left(\varphi_{2}\right)$, we have:

$$
\begin{aligned}
\left\langle S_{f, \omega}, \frac{1}{2} L_{X}(g)\right\rangle \geq & r \frac{\partial \log \varphi}{\partial r}\left[m f_{\omega}-f_{\omega}^{\prime} p|\omega|^{2}\right]+\frac{1}{2} f_{\omega}\left[(m-1) \lambda_{\min }+2\right] \\
& -\frac{1}{2} \max \left(2, \lambda_{\max }\right) f_{\omega}^{\prime} p|\omega|^{2} \\
\geq & \left(m r \frac{\partial \log \varphi}{\partial r}+\frac{m-1}{2} \lambda_{\min }+1\right) f_{\omega} \\
& -\left(2 p r \frac{\partial \log \varphi}{\partial r}+p \max \left(2, \lambda_{\max }\right)\right) f_{\omega}^{\prime} \frac{|\omega|^{2}}{2}
\end{aligned}
$$


by using the condition $s \frac{\partial f}{\partial s} \leq f$, we have $f_{\omega}^{\prime} \frac{|\omega|^{2}}{2} \leq f_{\omega}$, then:

$$
\begin{aligned}
\left\langle S_{f, \omega}, \frac{1}{2} L_{X}(g)\right\rangle & \geq\left[(m-2 p) r \frac{\partial \log \varphi}{\partial r}+\frac{m-1}{2} \lambda_{\min }+1-p \max \left(2, \lambda_{\max }\right)\right] f_{\omega} \\
& \geq C_{0} f_{\omega} .
\end{aligned}
$$

On the other hand, by the Coarea formula and $|\nabla r|_{g}=\varphi^{-1}$, we have:

$$
\begin{aligned}
\int_{\partial B(r)} S_{f, \omega}(X, \nu) d s_{g} & =\int_{\partial B(r)}\left[f_{\omega} g(X, \nu)-f_{\omega}^{\prime}(\omega \odot \omega)(X, \nu)\right] d s_{g} \\
& =\int_{\partial B(r)}\left[f_{\omega} g\left(r \frac{\partial}{\partial r}, \varphi^{-1} \partial r\right)-f_{\omega}^{\prime}(\omega \odot \omega)\left(r \frac{\partial}{\partial r}, \varphi^{-1} \partial r\right)\right] d s_{g} \\
& =r \int_{\partial B(r)} f_{\omega} \varphi d s_{g}-\int_{\partial B(r)} f_{\omega}^{\prime} r \varphi^{-1}(\omega \odot \omega)(\partial r, \partial r) d s_{g} \\
& \leq r \int_{\partial B(r)} f_{\omega} \varphi d s_{g} \\
& \leq r \frac{d}{d r} \int_{0}^{r}\left(\int_{\partial B(r)} \frac{f_{\omega}}{|\nabla r|} d s_{g}\right) d t \\
& \leq r \frac{d}{d r} \int_{\partial B(r)} f_{\omega} d v_{g} .
\end{aligned}
$$

If $\omega$ satisfies a generalized $f$-conservation law, then from (10), (18) and (17), we obtain:

$$
C_{0} \int_{B(r)} f_{\omega} d v_{g}+\int_{B(r)} r\left(d f\left(\frac{\partial}{\partial r}\right)\right)_{\omega} d v_{g} \leq r \frac{d}{d r} \int_{B(r)} f_{\omega} d v_{g}
$$

On the other hand, we have

$$
\begin{aligned}
\mu & =\sup _{M \times \mathbb{R}_{+}^{*}} r\left|\frac{\partial \log f}{\partial r}\right| \\
& =\sup _{M \times \mathbb{R}_{+}^{*}} \frac{r}{f}\left|\frac{\partial f}{\partial r}\right|,
\end{aligned}
$$

then $\mu \geq-\frac{r}{f_{\omega}}\left(\frac{\partial f}{\partial r}\right)_{\omega}$, so that $\mu \frac{f_{\omega}}{r} \geq-\left(\frac{\partial f}{\partial r}\right)_{\omega}$, and:

$$
\mu \int_{B(r)} f_{\omega} d v_{g} \geq-\int_{B(r)}\left(r \frac{\partial f}{\partial r}\right)_{\omega} d v_{g}
$$

we get:

$$
C_{0} \int_{B(r)} f_{\omega} d v_{g}-\mu \int_{B(r)} f_{\omega} d v_{g} \leq r \frac{d}{d r} \int_{B(r)} f_{\omega} d v_{g}
$$

that is:

$$
\left(C_{0}-\mu\right) \int_{B(r)} f_{\omega} d v_{g} \leq r \frac{d}{d r} \int_{B(r)} f_{\omega} d v_{g}
$$


so that:

$$
\frac{d}{d r} \frac{\int_{B(r)} f_{\omega} d v_{g}}{r^{C_{0}-\mu}} \geq 0
$$

Therefore,

$$
\frac{\int_{B\left(\rho_{1}\right)} f_{\omega} d v_{g}}{\rho_{1}^{C_{0}-\mu}} \leq \frac{\int_{B\left(\rho_{2}\right)} f_{\omega} d v_{g}}{\rho_{2}^{C_{0}-\mu}},
$$

for any $0<\rho_{1}<\rho_{2}$. This proves Theorem (1).

Let $u: M \longrightarrow N$ be an $f$-harmonic map. Then its differential du can be viewed as a 1-form with values in the induced bundle $u^{-1} T N$. Since $\omega=d u$ satisfies the generalized conservation law (see [11]), we obtain the following Liouville-type result:

Corollary 1. Suppose that $u:\left(M, \varphi^{2} g_{0}\right) \longrightarrow N$ is an $f$-harmonic map. If $C_{0}-\mu>0, \varphi$ satisfies $\left(\varphi_{1}\right),\left(\varphi_{2}\right)$ with $s \frac{\partial f}{\partial s} \leq f$ and $\frac{\partial f}{\partial s} \geq 0$, then

$$
\frac{\int_{B\left(\rho_{1}\right)} f_{u} d v_{g}}{\rho_{1}^{C_{0}-\mu}} \leq \frac{\int_{B\left(\rho_{2}\right)} f_{u} d v_{g}}{\rho_{2}^{C_{0}-\mu}}
$$

for any $0<\rho_{1}<\rho_{2}$, where $f_{u} \in C^{\infty}(M)$ defined by:

$$
f_{u}(x)=f\left(x, \frac{|d u|^{2}}{2}\right), \forall x \in M .
$$

Proof. This follows at once from Theorem 1 in which $p=1$ and $\omega=d u$.

From (19), we immediately get the following:

Corollary 2. Suppose that $\omega \in A^{p}(\xi),(p \geq 1)$ satisfies the generalized $f$ conservation law, and $\xi: E \longrightarrow(M, g)$ is a smooth Riemannian vector bundle over $\left(M, \varphi^{2} g_{0}\right)$. If $\varphi$ satisfies $\left(\varphi_{1}\right),\left(\varphi_{2}\right)$ and $f$ satisfies $s \frac{\partial f}{\partial s} \leq f, \frac{\partial f}{\partial s} \geq 0$, and $\frac{\partial f}{\partial r} \geq 0$, then

$$
\frac{\int_{B\left(\rho_{1}\right)} f_{u} d v_{g}}{\rho_{1}^{C_{0}}} \leq \frac{\int_{B\left(\rho_{2}\right)} f_{u} d v_{g}}{\rho_{2}^{C_{0}}},
$$

for any $0<\rho_{1}<\rho_{2}$.

Lemma 1. ([g]) Let $(M, g)$ be a complete Riemannian manifold with a pole $x_{0}$. Denote by $K_{r}$ the radial curvature of $M$.

i) If $-\alpha^{2} \leq K_{r} \leq-\beta^{2}$ with $\alpha \geq \beta>0$ and $(m-1) \beta-2 p \alpha \geq 0$, then:

$$
\left[(m-1) \lambda_{\min }+2 p \max \left(2, \lambda_{\max }\right)\right] \geq 2\left(m-\frac{2 p \alpha}{\beta}\right) .
$$

ii) If $-\frac{A}{\left(1+r^{2}\right)^{1+\epsilon}} \leq K_{r} \leq \frac{B}{\left(1+r^{2}\right)^{1+\epsilon}}$ with $\epsilon>0, A>0$ and $0 \leq B \leq 2 \epsilon$, then:

$$
\left[(m-1) \lambda_{\min }+2 p \max \left(2, \lambda_{\max }\right)\right] \geq 2\left[1+(m-1)\left(1-\frac{B}{2 \epsilon}\right)-2 p e^{\frac{A}{2 \epsilon}}\right] .
$$


iii) If $-\frac{a^{2}}{c^{2}+r^{2}} \leq K_{r} \leq \frac{b^{2}}{c^{2}+r^{2}}$ with $a \geq 0, b^{2} \in\left[0, \frac{1}{4}\right]$ and $c^{2} \geq 0$, then:

$$
\left[(m-1) \lambda_{\min }+2 p \max \left(2, \lambda_{\max }\right)\right] \geq 2\left[1+(m-1) \frac{1+\sqrt{1-4 b^{2}}}{2}-2 p \frac{1+\sqrt{1+4 a^{2}}}{2}\right] .
$$

Theorem 2. Let $(M, g)$ be an $m$-dimensional complete manifold with a pole $x_{0}$. Assume that the radial curvature $K_{r}$ of $M$ satisfies one of the following three conditions:

i) $-\alpha^{2} \leq K_{r} \leq-\beta^{2}$ with $\alpha \geq \beta>0$ and $(m-1) \beta-2 p \alpha \geq 0$;

ii) $-\frac{A}{\left(1+r^{2}\right)^{1+\epsilon}} \leq K_{r} \leq \frac{B}{\left(1+r^{2}\right)^{1+\epsilon}}$ with $\epsilon>0, A>0$ and $0 \leq B \leq 2 \epsilon$;

iii) $-\frac{a^{2}}{c^{2}+r^{2}} \leq K_{r} \leq \frac{b^{2}}{c^{2}+r^{2}}$ with $a \geq 0, b^{2} \in\left[0, \frac{1}{4}\right]$ and $c^{2} \geq 0$.

If $\omega \in A^{p}(\xi),(p \geq 1)$ satisfies the generalized $f$-conservation law with $s \frac{\partial f}{\partial s} \leq f$, $\frac{\partial f}{\partial s} \geq 0$ and $\Lambda-\mu>0$, then

$$
\frac{\int_{B\left(\rho_{1}\right)} f_{\omega} d v_{g}}{\rho_{1}^{\Lambda-\mu}} \leq \frac{\int_{B\left(\rho_{2}\right)} f_{\omega} d v_{g}}{\rho_{2}^{\Lambda-\mu}},
$$

for any $0<\rho_{1}<\rho_{2}$, where:

$$
\Lambda=\left\{\begin{array}{l}
\left.m-\frac{2 p \alpha}{\beta}, \text { if } K_{r} \text { satisfies } i\right) \\
\left.1+(m-1)\left(1-\frac{\beta}{2 \epsilon}\right)-2 p e^{\frac{A}{2 \epsilon}}, \text { if } K_{r} \text { satisfies } \text { ii }\right) \\
1+(m-1) \frac{1+\sqrt{1-4 b^{2}}}{2}-2 p \frac{1+\sqrt{1+4 a^{2}}}{2}, \text { if } K_{r} \text { satisfies } \text { iii) } .
\end{array}\right.
$$

Proof. From the proof of Theorem 1 for $\varphi=1$ and Lemma 1, we have

$$
\frac{d}{d r} \frac{\int_{B(r)} f_{\omega} d v_{g}}{r^{\Lambda-\mu}} \geq 0
$$

therefore, we get the monotonicity formula:

$$
\frac{\int_{B\left(\rho_{1}\right)} f_{\omega} d v_{g}}{\rho_{1}^{\Lambda-\mu}} \leq \frac{\int_{B\left(\rho_{2}\right)} f_{\omega} d v_{g}}{\rho_{2}^{\Lambda-\mu}},
$$

for any $0<\rho_{1}<\rho_{2}$.

Corollary 3. Let $M, K_{r}$ and $\Lambda$ be as in Theorem 2. If $\omega \in A^{p}(\xi),(p \geq 1)$ satisfies the generalized $f$-conservation law, with $s \frac{\partial f}{\partial s} \leq f, \frac{\partial f}{\partial s} \geq 0$ and $\frac{\partial f}{\partial r} \geq 0$, then

$$
\frac{\int_{B\left(\rho_{1}\right)} f_{\omega} d v_{g}}{\rho_{1}^{\Lambda}} \leq \frac{\int_{B\left(\rho_{2}\right)} f_{\omega} d v_{g}}{\rho_{2}^{\Lambda}},
$$

for any $0<\rho_{1}<\rho_{2}$.

Proof. from Corollary (2) for $\varphi=1$, we know that formula (20) is true. 
From [9], we can say that the functional $E_{f}(\omega)$ of $\omega \in A^{p}(\xi)$ is slowly divergent if there exists a positive function $\psi(r)$ with $\int_{R_{0}}^{\infty} \frac{d r}{r \psi(r)}=\infty\left(R_{0}>0\right)$, such that:

$$
\lim _{R \rightarrow \infty} \int_{B(R)} \frac{f_{\omega}}{\psi(r(x))} d v_{g}<\infty
$$

Theorem 3. Suppose that $\omega \in A^{p}(\xi)(p \geq 1)$ satisfies the following equation:

$$
\int_{M}\left(\operatorname{div} S_{f, \omega}\right)(X) d v_{g}=\int_{M} X(f)_{\omega} d v_{g}
$$

for any $X \in \Gamma(T M)$, and $\xi: E \longrightarrow(M, g)$ is a smooth Riemannian vector bundle $\operatorname{over}\left(M, \varphi^{2} g_{0}\right)$. If $C_{0}-\mu>0, \varphi$ satisfies $\left(\varphi_{1}\right),\left(\varphi_{2}\right)$ and $E_{f}(\omega)$ is slowly divergent with $s \frac{\partial f}{\partial s} \leq f$ and $\frac{\partial f}{\partial s} \geq 0$, then $\omega=0$.

Proof. From (17), we have:

$$
\left\langle S_{f, \omega}, \frac{1}{2} L_{X} g\right\rangle+(X(f))_{\omega} \geq\left(C_{0}-\mu\right) f_{\omega}
$$

On the other hand, taking $D=B(r)$ and $T=S_{f, \omega}$ in (7) of [9], we have

$$
\int_{\partial D} S_{f, \omega}(X, \nu) d s_{g}=\int_{D}\left[\left\langle S_{f, \omega}, \frac{1}{2} L_{X} g\right\rangle+\left(\operatorname{div} S_{f, \omega}\right)(X)\right] d v_{g}
$$

so that:

$$
\begin{aligned}
& \int_{B(r)}\left\langle S_{f, \omega}, \frac{1}{2} L_{X} g\right\rangle d v_{g}+\int_{B(r)}\left(\operatorname{div} S_{f, \omega}\right)(X) d v_{g} \\
= & \int_{\partial B(r)} S_{f, \omega}(X, \nu) d s_{g} \\
= & \int_{\partial B(r)} f_{\omega} g(X, \nu) d s_{g}-\int_{\partial B(r)} f_{\omega}^{\prime}(\omega \odot \omega)(X, \nu) d s_{g} \\
= & \left.r \int_{\partial B(r)} f_{\omega} \varphi d s_{g}-\int_{\partial B(r)} f_{\omega}^{\prime} \varphi^{-1} r \sum_{i=1}^{m}<i_{\frac{\partial}{\partial r}} \omega, i_{\frac{\partial}{\partial r}} \omega\right) d s_{g} \\
\leq & r \int_{\partial B(r)} f_{\omega} \varphi d s_{g} .
\end{aligned}
$$

Here $f_{\omega}^{\prime} \geq 0$ because $\frac{\partial f}{\partial s} \geq 0$. Now suppose that $\omega$ is not always zero, so there exists a constant $R_{1}>0$ such that for $R \geq R_{1}$,

$$
\int_{B(R)} f_{\omega} d v_{g} \geq C_{3}, C_{3}>0 .
$$

From (22):

$$
\lim _{R \rightarrow \infty} \int_{B(R)}\left(\operatorname{div} S_{f, \omega}\right)(X) d v_{g}=\lim _{R \rightarrow \infty} \int_{B(R)}(X(f))_{\omega} d v_{g}
$$


then, $\exists R_{2}>R_{1}$ such that $\forall R \geq R_{2}$ :

$$
-\frac{\left(C_{0}-\mu\right)}{2} C_{3} \leq \int_{B(R)}\left(\operatorname{div} S_{f, \omega}\right)(X) d v_{g}-\int_{B(R)} X(f)_{\omega} d v_{g} \leq \frac{\left(C_{0}-\mu\right)}{2} C_{3}
$$

from (23), (24) and (26), we obtain

$$
\begin{aligned}
R \int_{\partial B(R)} f_{\omega} \varphi d s_{g} & \geq \int_{B(R)}<S_{f, \omega}, \frac{1}{2} L_{X} g>d v_{g}+\int_{B(R)}\left(\operatorname{div} S_{f, \omega}\right)(X) d v_{g} \\
& \geq \int_{B(R)}\left[<S_{f, \omega}, \frac{1}{2} L_{X} g>+X(f)_{\omega}\right] d v_{g}-\frac{\left(C_{0}-\mu\right)}{2} C_{3} \\
& \geq\left(C_{0}-\mu\right) \int_{B(R)} f_{\omega} d v_{g}-\frac{\left(C_{0}-\mu\right)}{2} C_{3} \\
& \geq \frac{\left(C_{0}-\mu\right)}{2} C_{3} .
\end{aligned}
$$

that is:

$$
\int_{\partial B(R)} f_{\omega} \varphi d s_{g} \geq \frac{\left(C_{0}-\mu\right)}{2 R} C_{3}
$$

From $(27)$ and $|\nabla r|=\varphi^{-1}$, we have:

$$
\begin{aligned}
\lim _{R \rightarrow \infty} \int_{B(R)} \frac{f_{\omega}}{\psi(r(x))} d v_{g} & =\int_{0}^{\infty} \frac{d R}{\psi(R)} \int_{\partial B(R)} f_{\omega} \varphi d s_{g} \\
& \geq \int_{R_{2}}^{\infty} \frac{d R}{\psi(R)} \int_{\partial B(R)} f_{\omega} \varphi d s_{g} \\
& \geq \int_{R_{2}}^{\infty} \frac{\left(C_{0}-\mu\right)}{2 R} C_{3} \frac{d R}{\psi(R)} \\
& \geq\left(C_{0}-\mu\right) C_{3} \int_{R_{2}}^{\infty} \frac{d R}{2 R \psi(r)}=\infty
\end{aligned}
$$

which contradicts $(21)$, therefore $\omega \equiv 0$

Theorem 4. Suppose that $\omega \in A^{p}(\xi)(p \geq 1)$, satisfies the generalized integral $f$-conservation law, i.e.

$$
\int_{M}\left(\operatorname{div} S_{f, \omega}\right)(X) d v_{g}=\int_{M} X(f)_{\omega} d v_{g}
$$

and $\xi: E \longrightarrow(M, g)$ is a smooth Riemannian vector bundle over $\left(M, \varphi^{2} g_{0}\right)$. If $C_{0}-\mu>0, \varphi$ satisfies $\left(\varphi_{1}\right)$ and $\left(\varphi_{2}\right)$, with $\int_{M} f_{\omega} d v_{g}<\infty$ and $s \frac{\partial f}{\partial s} \leq f$, then $\omega \equiv 0$.

Proof. We take $X=\phi(r) r \frac{\partial}{\partial r}=\frac{1}{2} \phi(r) \nabla^{0} r^{2}$, where $\nabla^{0}$ denote the covariant derivative determined by $g_{0}$ and $\phi(r)$ is a nonnegative function determined later. By a direct computation, we have:

$$
\left\langle S_{f, \omega}, \frac{1}{2} L_{X} g\right\rangle=\phi(r) r \frac{\partial \log \varphi}{\partial r}\left\langle S_{f, \omega}, g\right\rangle+\frac{1}{2} \varphi^{2}\left\langle S_{f, \omega}, L_{\phi(r) r \frac{\partial}{\partial r}} g_{0}\right\rangle .
$$


Let $\left\{e_{i}\right\}_{i=1}^{m}$ be an orthonormal basis with respect to $g_{0}$ and $e_{m}=\frac{\partial}{\partial r}$. We may assume that $\mathrm{Hess}_{g_{0}}\left(r^{2}\right)$ becomes a diagonal matrix with respect to $\left\{e_{i}\right\}_{i=1}^{m}$. Then $\left\{\tilde{e}_{i}=\varphi^{-1} e_{i}\right\}$ is an orthonormal basis with respect to $g$. We compute:

$$
\begin{aligned}
\varphi^{2}\left\langle S_{f, \omega}, \frac{1}{2} L_{\phi(r) r \frac{\partial}{\partial r}} g_{0}\right\rangle= & \varphi^{2} \sum_{i, j=1}^{m} S_{f, \omega}\left(\tilde{e}_{i}, \tilde{e}_{j}\right)\left(L_{\phi(r) r \frac{\partial}{\partial r}} g_{0}\right)\left(\tilde{e}_{i}, \tilde{e}_{j}\right) \\
= & \varphi^{2}\left[\sum_{i, j=1}^{m} f_{\omega} g\left(\tilde{e}_{i}, \tilde{e}_{j}\right)\left(L_{\phi(r) r \frac{\partial}{\partial r}} g_{0}\right)\left(\tilde{e}_{i}, \tilde{e}_{j}\right)\right. \\
& \left.-f_{\omega}^{\prime}(\omega \odot \omega)\left(\tilde{e}_{i}, \tilde{e}_{j}\right)\left(L_{\phi(r) r \frac{\partial}{\partial r}} g_{0}\right)\left(\tilde{e}_{i}, \tilde{e}_{j}\right)\right] \\
= & \sum_{i=1}^{m} f_{\omega}\left(L_{\phi}(r) r \frac{\partial}{\partial r} g_{0}\right)\left(e_{i}, e_{i}\right) \\
& -\sum_{i, j=1}^{m} f_{\omega}^{\prime}(\omega \odot \omega)\left(\tilde{e}_{i}, \tilde{e}_{j}\right)\left(L_{\phi(r) r} \frac{\partial}{\partial r} g_{0}\right)\left(e_{i}, e_{j}\right) \\
= & \phi(r) \sum_{i=1}^{m} f_{\omega} H e s s_{g_{0}}\left(r^{2}\right)\left(e_{i}, e_{i}\right)+2 f_{\omega} r \phi^{\prime}(r) \\
& -\phi(r) \sum_{i, j=1}^{m} f_{\omega}^{\prime}(\omega \odot \omega)\left(\tilde{e}_{i}, \tilde{e}_{j}\right) H e s s_{g_{0}}\left(r^{2}\right)\left(e_{i}, e_{j}\right) \\
& -2 f_{\omega}^{\prime} r \phi^{\prime}(r)(\omega \odot \omega)\left(\tilde{e}_{m}, \tilde{e}_{m}\right) \\
\geq & \phi(r) f_{\omega}\left[2+(m-1) \lambda_{\min }\right] \\
& -\phi(r) f_{\omega}^{\prime} \max \left(2, \lambda_{\max }\right) \sum_{i=1}^{m-1}(\omega \odot \omega)\left(\tilde{e}_{i}, \tilde{e}_{i}\right) \\
& +2 f_{\omega} r \phi^{\prime}(r)-2 f_{\omega}^{\prime} r \phi^{\prime}(r)(\omega \odot \omega)\left(\tilde{e}_{m}, \tilde{e}_{m}\right) \\
\geq & \phi(r) f_{\omega}\left[2+(m-1) \lambda_{\min }\right] \\
& -\phi(r) f_{\omega}^{\prime} \max \left(2, \lambda_{\max }\right) p|\omega|^{2} 2 f_{\omega} r \phi^{\prime}(r) \\
& -2 f_{\omega}^{\prime} r \phi^{\prime}(r)(\omega \odot \omega)\left(\tilde{e}_{m}, \tilde{e}_{m}\right) . \\
& \\
& \\
&
\end{aligned}
$$

As $s \frac{\partial f}{\partial s} \leq f$ we find that $\frac{|\omega|^{2}}{2} f_{\omega}^{\prime} \leq f_{\omega}$, we obtain:

$$
\begin{aligned}
\varphi^{2}\left\langle S_{f, \omega}, \frac{1}{2} L_{\phi(r) r \frac{\partial}{\partial r}} g_{0}\right\rangle \geq & \phi(r) f_{\omega}\left[2+(m-1) \lambda_{\min }\right. \\
& \left.-2 p \max \left(2, \lambda_{\max }\right)\right]+2 f_{\omega} r \phi^{\prime}(r) \\
& -2 f_{\omega}^{\prime} r \phi^{\prime}(r)(\omega \odot \omega)\left(\tilde{e}_{m}, \tilde{e}_{m}\right) .
\end{aligned}
$$

From $(14),(28),(29)$ and $\left(\varphi_{1}\right),\left(\varphi_{2}\right)$ :

$$
\begin{aligned}
\left\langle S_{f, \omega}, \frac{1}{2} L_{X} g\right\rangle \geq & \phi(r) r \frac{\partial \log \varphi}{\partial r}\left[m f_{\omega}-2 p f_{\omega}\right]+\phi(r) f_{\omega}\left[1+\frac{(m-1)}{2} \lambda_{\min }-p \max \left(2, \lambda_{\max }\right)\right] \\
& +f_{\omega} r \phi^{\prime}(r)-f_{\omega}^{\prime} r \phi^{\prime}(r)(\omega \odot \omega)\left(\tilde{e}_{m}, \tilde{e}_{m}\right)
\end{aligned}
$$




$$
\begin{aligned}
\geq & \phi(r) r \frac{\partial \log \varphi}{\partial r} f_{\omega}[m-2 p]+\phi(r) f_{\omega}\left[1+\frac{(m-1)}{2} \lambda_{\min }-p \max \left(2, \lambda_{\max }\right)\right] \\
& +f_{\omega} r \phi^{\prime}(r)-f_{\omega}^{\prime} r \phi^{\prime}(r)(\omega \odot \omega)\left(\tilde{e}_{m}, \tilde{e}_{m}\right) \\
\geq & \phi(r) f_{\omega}\left[(m-2 p) r \frac{\partial \log \varphi}{\partial r}+1+\frac{(m-1)}{2} \lambda_{\min }-p \max \left(2, \lambda_{\max }\right)\right] \\
& +f_{\omega} r \phi^{\prime}(r)-f_{\omega}^{\prime} r \phi^{\prime}(r)(\omega \odot \omega)\left(\tilde{e}_{m}, \tilde{e}_{m}\right) \\
\geq & C_{0} \phi(r) f_{\omega}+f_{\omega} r \phi^{\prime}(r)-f_{\omega}^{\prime} r \phi^{\prime}(r)(\omega \odot \omega)\left(\tilde{e}_{m}, \tilde{e}_{m}\right) .
\end{aligned}
$$

From $\mu=\sup _{M \times \mathbb{R}_{+}^{*}} \frac{r}{f}\left|\frac{\partial f}{\partial r}\right|$, we have:

$$
\mu \geq-r \frac{1}{f(x, s)}\left(\frac{\partial f}{\partial r}\right)_{(x, s)}, \forall(x, s) \in M \times \mathbb{R}
$$

so that:

$$
\mu \geq-r \frac{1}{f_{\omega}}\left(\frac{\partial f}{\partial r}\right)_{\omega} \frac{\phi(r)}{\phi(r)}
$$

that is:

$$
X(f)_{\omega} \geq-\mu f_{\omega} \phi(r)
$$

We get:

$$
\left\langle S_{f, \omega}, \frac{1}{2} L_{X} g\right\rangle+X(f)_{\omega} \geq\left(C_{0}-\mu\right) \phi(r) f_{\omega}+f_{\omega} r \phi^{\prime}(r)-f_{\omega}^{\prime} r \phi^{\prime}(r)(\omega \odot \omega)\left(\tilde{e}_{m}, \tilde{e}_{m}\right) .
$$

For any fixed $R>0$, we take a smooth function $\phi(r)$ which takes value 1 on $B_{\frac{R}{2}}\left(x_{0}\right), 0$ outside $B_{R}\left(x_{0}\right)$ and $0 \leq \phi(r) \leq 1$ on $T_{R}\left(x_{0}\right)=B_{R}\left(x_{0}\right)-B_{\frac{R}{2}}\left(x_{0}\right)$. And $\phi(r)$ also satisfies the condition: $\left|\phi^{\prime}(r)\right| \leq \frac{C_{2}}{r}$ on M, where $C_{2}$ is a positive constant.

From (12) and (31), with the condition $s \frac{\partial f}{\partial s} \leq f$, we have:

$$
\begin{aligned}
0 & \geq \int_{M}\left(C_{0}-\mu\right) \phi(r) f_{\omega} d v_{g}+\int_{M} f_{\omega} r \phi^{\prime}(r) d v_{g}-\int_{M} f_{\omega}^{\prime} r \phi^{\prime}(r)(\omega \odot \omega)\left(\tilde{e}_{m}, \tilde{e}_{m}\right) d v_{g} \\
& \geq \int_{B_{\frac{R}{2}}\left(x_{0}\right)}\left(C_{0}-\mu\right) f_{\omega} d v_{g}+\int_{T_{R}\left(x_{0}\right)} f_{\omega} r \phi^{\prime}(r) d v_{g}-\int_{T_{R}\left(x_{0}\right)} f_{\omega}^{\prime} r \phi^{\prime}(r)(\omega \odot \omega)\left(\tilde{e}_{m}, \tilde{e}_{m}\right) d v_{g} \\
& \geq \int_{B_{\frac{R}{2}}\left(x_{0}\right)}\left(C_{0}-\mu\right) f_{\omega} d v_{g}-C_{2} \int_{T_{R}\left(x_{0}\right)} f_{\omega} d v_{g}-C_{2} p \int_{T_{R}\left(x_{0}\right)} f_{\omega}^{\prime}|\omega|^{2} d v_{g} \\
& \geq\left(C_{0}-\mu\right) \int_{B_{\frac{R}{2}}\left(x_{0}\right)} f_{\omega} d v_{g}-C_{2} \int_{T_{R}\left(x_{0}\right)} f_{\omega} d v_{g}-2 C_{2} p \int_{T_{R}\left(x_{0}\right)} f_{\omega} d v_{g} \\
& \geq\left(C_{0}-\mu\right) \int_{B_{\frac{R}{2}}\left(x_{0}\right)} f_{\omega} d v_{g}-(1+2 p) C_{2} \int_{T_{R}\left(x_{0}\right)} f_{\omega} d v_{g} .
\end{aligned}
$$

From $\int_{M} f_{\omega} d v_{g}<\infty$, we obtain $\lim _{R \longrightarrow \infty} \int_{T_{R}\left(x_{0}\right)} f_{\omega} d v_{g}=0$, so by equation (32), we have:

$$
0 \geq\left(C_{0}-\mu\right) \lim _{R \longrightarrow \infty} \int_{B_{\frac{R}{2}}\left(x_{0}\right)} f_{\omega} d v_{g}
$$


that is:

$$
0 \geq\left(C_{0}-\mu\right) \int_{M} f_{\omega} d v_{g}
$$

So that $\omega=0$.

\section{Acknowledgements:}

The authors would like to thank Professor Mustapha Djaa for his assistance in the idea of this work and its guidelines.

This note was supported by L.G.A.C.A. Laboratory of Saida university and Algerian agency P.R.F.U project.

\section{References}

[1] Baird, P., Stress-energy tensors and the Lichnerowicz Laplacian, J. Geom. Phys. 58(2008), no. 10, 1329-1342.

[2] Baird, P. and Eells, J., A conservation law for harmonic maps, In: Geometry Symposium Utrecht 1980 (Looijenga, E., Siersma, D. and Takens, F. eds.), Lecture Notes in Mathematics, Vol. 894, Berlin, Springer-Verlag, 1981, 1-25.

[3] Chiang, Y.J., f-Biharmonic maps between Riemannian manifolds, Department of Mathematics, University of Mary Washington Fredericksburg, VA 22401, USA 2012.

[4] Djaa, M. and Cherif, A.M., f-Harmonic maps and Liouville type theorem, Konuralp J. Math. 4 (2016), no. 1, 33-44.

[5] Djaa, M. and Cherif, A.M., On generalized $f$-biharmonic maps and stress f-bienergy tensor, J. Geom. Symmetry Phys. 29 (2013), 65-81.

[6] Djaa, M., Cherif, A.M., Zagga, K. and Ouakkas, S., On the generalized of harmonic and bi-harmonic maps, Int. Electronic J. Geom. 5 (2012), no. 1, $90-100$.

[7] Dong, Y.X. and Wei, S.W., On vanishing theorems for vector bundle valued p-forms and their applications, Comm. Math. Phys. 304 (2011), no. 2, 329368.

[8] Feng, S., and Han, Y., Liouville type theorems of f-Harmonic maps with potential, Results Math. 66 (2014), no. 1-2, 43-64.

[9] Han, Y., and Feng, S., Monotonicity formulas and vanishing theorems for vector bundle valued p-forms, Advance in Mathematics 4 (2015), 1-12. 
[10] Lu, W.J., On f-bi-harmonic maps and bi-f-harmonic maps between Riemannian manifolds. Sci. China Math. Springer, 58 (2015), 1483-1498.

[11] Ouakkas, S., Nasri, R. and Djaa, M., On the f-harmonic and f-biharmonic maps, JP J. Geom. Topol., 10 (2010), no. 1, 11-27.

[12] Ou, Y.L., On f-harmonic morphisms between Riemannian manifolds, Chinese ANN. Math. Ser. B, 35 (2014), 225-236.

[13] Sealey, H.C.J., The stress energy tensor and vanishing of L2 harmonic forms, preprint. 